\title{
Pressure effects on bimolecular recombination and unimolecular dissociation reactions
}

\author{
R. A. Marcus ${ }^{\mathrm{a})}$ and Yi Qin Gao \\ Noyes Laboratory of Chemical Physics, California Institute of Technology, Pasadena, California 91125
}

(Received 22 January 2001; accepted 19 March 2001)

\begin{abstract}
The treatment of pressure effects on bimolecular recombinations and unimolecular dissociations is discussed. The analysis of recombination and dissociation reactions is made by showing how the nonequilibrium energy $(E)$ and angular momentum $(J)$-dependent steady-state population distribution functions for the two reactions are related to each other and to the equilibrium population distribution function at the given $E$ and $J$. As a special case a strong collision model is then used for the collisional rotational angular momentum transfer, and a ladder model for the collisional energy transfer. An analytical result is obtained for states below the dissociation threshold. The extension to recombinations with two exit channels is described, for application to ozone formation and isotopic effects. (c) 2001 American Institute of Physics.
\end{abstract}

[DOI: $10.1063 / 1.1370552]$

\section{INTRODUCTION}

Unimolecular dissociations and bimolecular associations have been intensively studied in the literature, and have been treated in well-known texts, monographs, and articles. ${ }^{1-5}$ It has been recognized for many years that weak collisions play a significant role in the activation-deactivation process and are needed to explain the detailed experimental results on pressure effects. ${ }^{1,2,6-8}$ An important quantity in these collisions is the average energy of transfer $\Delta E,\langle\Delta E\rangle$, for the deactivating collisions and, determined by microscopic reversibility, the average energy transfer in upward (activating) collisions. Studies have revealed that apart from rare "supercollisions" "9-11 the results for the energy transfer depend mainly on $\langle\Delta E\rangle$ (and the microscopic reversibility related upward average), and are relatively insensitive to the precise form of the collisional energy transfer function $\omega\left(E \rightarrow E^{\prime}\right) .^{1,2}$ In a recent study we have noted that an unusual "mass-independent" effect in recombination rates in "scrambled" systems and unconventional "massdependent'" effects in unscrambled ones, may, in the interpretation given there, provide information on the dependence of $\langle\Delta E\rangle$ on temperature. ${ }^{12}$ We discuss this particular aspect elsewhere, ${ }^{13}$ using the formalism in the present paper, as well as using an alternative "exponential-down" model for the collisional deactivation. ${ }^{2}$ These applications of the present paper are to recombinations, ${ }^{14}$ to their isotopic effects, ${ }^{15,16}$ and to isotopic exchange reactions. ${ }^{17}$

The formalism is first given in Sec. II for the case of one distinguishable exit channel, i.e., for a system $X+Y X$ $\rightarrow X Y X^{*} \rightarrow X Y+X$, the asterisk denoting an energetic molecule. In Sec. II the relation of the steady-state distribution functions, as a function of the energy $E$ and angular momentum $J$, is described for the recombination and dissociation reactions, both to each other and to the equilibrium distribution function. The treatment is specialized in Sec. III to in-

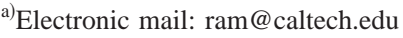

clude the strong collision approximation for rotations and a stepladder model for the collisional energy transfer, so leading to an analytical expression for the diffusion in "energy space" below the reaction threshold for dissociation. This model reduces significantly the number of quantum states needed in a numerical treatment. In Sec. IV the treatment is extended to the case of two distinguishable exit channels, e.g., $\quad X+Y Z \rightarrow X Y Z^{*} \rightarrow X+Y Z$ or $X Y+Z(X \neq Z)$. The present equations, or their equivalent, are utilized elsewhere. ${ }^{13}$ Isotopic exchange reactions are discussed in Sec. V.

\section{THEORETICAL CONSIDERATIONS}

\section{A. Recombination}

For the recombination of two particles $X$ and $Y Z$, where $X$ may denote an atom or larger particle and $Y Z$ may denote a diatomic or larger particle, the reaction sequence for recombination can be written as

$$
\begin{aligned}
& X+Y Z \stackrel{k_{a}^{\prime}(E J)}{\longrightarrow} X Y Z(E J), \\
& X Y Z(E J) \stackrel{\omega\left(E \rightarrow E^{\prime}, J \rightarrow J^{\prime}\right)}{\longrightarrow} X Y Z\left(E^{\prime} J^{\prime}\right), \\
& X Y Z(E J) \stackrel{k_{a}(E J)}{\longrightarrow} X+Y Z, \\
& X Y Z(E J) \stackrel{k_{b}(E J)}{\longrightarrow} X Y+Z,
\end{aligned}
$$

where the energy $E$ and the total angular momentum $J$ of the colliding particles $X$ and $Y Z$ are constants of motion in Eqs. (2.1), (2.3), and (2.4), and where, depending on (EJ), either or both $k_{a}(E J)$ and $k_{b}(E J)$ may vanish. Reactions (2.3) and (2.4) tacitly include $X Y Z\left(E^{\prime} J^{\prime}\right)$ 's since the $E J$ in Eq. (2.1) can be an $E^{\prime} J^{\prime}$ and the double integration later in Eqs. (2.7) and (2.13) is over all $E, J, E^{\prime}$, and $J^{\prime}$. 
In addition to the first step (2.1) a different reaction product $X Z Y(E J)$ may also be formed, with its own subsequent sequence of collisional and dissociation steps. It can be treated separately. ${ }^{18,19}$

The collisional frequency $\omega\left(E \rightarrow E^{\prime}, J \rightarrow J^{\prime}\right)$ for $E$ and $J$ transfer is typically proportional to the concentration of the ambient gas. The component $K$ of the angular momentum of $X Y Z$ along a major symmetry or near-symmetry axis of $X Y Z$ may or may not be approximately constant during the lifetime of $X Y Z$. We shall consider the latter case ("active rotation" $K$ ), and integrate over $K$ in calculating the density of quantum states of an $X Y Z$ of given $E$ and $J$. We first consider the case where there is only one distinguishable exit channel, i.e., where $Z=X$ in Eqs. (2.1)-(2.4).

We let $c(E J)$ be the concentration of $X Y Z(E J)$ arising from reaction (2.1), abbreviate $\omega\left(E \rightarrow E^{\prime}, J \rightarrow J^{\prime}\right)$ by $\omega\left(E J, E^{\prime} J^{\prime}\right)$, and write

$$
\int_{E^{\prime}} \int_{J^{\prime}} \omega\left(E J, E^{\prime} J^{\prime}\right) d E^{\prime} d J^{\prime}=\omega
$$

where $\omega$ is the total collision frequency. We define a population distribution function $g(E J)$ :

$$
g(E J)=c(E J) /(X)(Y Z),
$$

where $(X)$ and $(Y Z)$ denote concentrations. In a steady-state approximation for $g(E J)$ we have ${ }^{20}$

$$
\begin{aligned}
0=\frac{d}{d t} g(E J)= & k_{a}^{\prime}(E J)-\left[k_{a}(E J)+\omega\right] g(E J) \\
& +\iint \omega\left(E^{\prime} J^{\prime}, E J\right) g\left(E^{\prime} J^{\prime}\right) d E^{\prime} d J^{\prime} .
\end{aligned}
$$

This equation can be rewritten by defining an equilibrium population distribution function $g_{\text {eq }}(E J)$, such that when introduced into Eq. (2.7) the forward and reverse reaction rate constants are equal

$$
k_{a}(E J) g_{\mathrm{eq}}(E J)=k_{a}^{\prime}(E J)
$$

(microscopic reversibility), while the collision terms are also equal (again, microscopic reversibility):

$$
\omega g_{\mathrm{eq}}(E J)=\iint \omega\left(E^{\prime} J^{\prime}, E J\right) g_{\mathrm{eq}}\left(E^{\prime} J^{\prime}\right) d E^{\prime} d J^{\prime} .
$$

Equation (2.7) then becomes

$$
\begin{aligned}
& {\left[k_{a}(E J)+\omega\right]\left[g_{\mathrm{eq}}(E J)-g(E J)\right]} \\
& \quad=\iint \omega\left(E^{\prime} J^{\prime}, E J\right)\left[g_{\mathrm{eq}}\left(E^{\prime} J^{\prime}\right)-g\left(E^{\prime} J^{\prime}\right)\right] d E^{\prime} d J^{\prime} .
\end{aligned}
$$

We denote the difference between the equilibrium population distribution function $g_{\mathrm{eq}}(E J)$ and $g(E J)$ by $f(E J)$,

$$
f(E J)=g_{\text {eq }}(E J)-g(E J) .
$$

Thereby, Eqs. (2.10) and (2.11) yield a "master equation" for $f(E J)$,

$\left[k_{a}(E J)+\omega\right] f(E J)=\iint \omega\left(E^{\prime} J^{\prime}, E J\right) f\left(E^{\prime} J^{\prime}\right) d E^{\prime} d J^{\prime}$.
The bimolecular recombination rate constant $k_{\mathrm{bi}}$ is given by

$$
k_{\mathrm{bi}}=\iint\left[k_{a}^{\prime}(E J)-g(E J) k_{a}(E J)\right] d E d J,
$$

which with Eqs. (2.8) and (2.11) yields

$$
k_{\mathrm{bi}}=\iint k_{a}(E J) f(E J) d E d J .
$$

The rate constant $k_{\mathrm{bi}}$ is calculated by solving Eq. (2.12) for $f(E J)$ and introducing it into Eq. (2.14), or alternatively by calculating the net collisional downward diffusion of the molecule $X Y Z$ in energy space to form a fully deactivated $X Y Z$ molecule.

\section{B. Unimolecular dissociation}

We denote by $C(E J)$ the concentration of energetically excited $X Y Z$ molecules arising in the unimolecular dissociation and introduce a population distribution function $F(E J)$ defined by

$$
F(E J)=C(E J) /(X Y Z),
$$

where $(X Y Z)$ is the concentration of $X Y Z$. The unimolecular dissociation rate is given by

$$
k_{\text {uni }}(X Y Z)=\iint k_{a}(E J) C(E J) d E d J
$$

and so

$$
k_{\text {uni }}=\iint k_{a}(E J) F(E J) d E d J .
$$

Before proceeding with the equations leading to the ana$\log$ of Eqs. (2.12) and (2.14) for unimolecular reactions we first note that when there is an equilibrium between $X+Y Z$ and $X Y Z$, the equilibrium concentration of excited $X Y Z$ at any $(E J), c_{\mathrm{eq}}(E J)$, is the sum of two terms:

$$
c_{\mathrm{eq}}(E J)=c(E J)+C(E J),
$$

where $c(E J)$ consists of marked $X Y Z$ molecules (labeled by a dagger, say) which originated from $X+Y Z$, and of differently marked $X Y Z$ molecules (labeled by two daggers) of concentration $C(E J)$, which originated from $X Y Z$. Each marked (dagger or two daggers) molecule may either continue on to the reactants or products region. This progress is followed only until it has reached either destination, at which point it loses its label. If it leaves again, it is with a mark appropriate to the new departure point, dagger or two daggers. That is, the equilibrium population $c_{\text {eq }}(E J)$ consists of molecules $X Y Z^{\dagger}$ which arose from $X+Y Z$ and molecules $X Y Z^{\dagger \dagger}$ which arose from $X Y Z$, but where neither of them has yet arrived with its current label at a destination. In this way we consider in the equilibrium population, $c_{\mathrm{eq}}(E J)$, the point of origin of the $X Y Z(E J)$ and so include in our considerations the forward and reverse reactions.

We have introduced expressions for the concentrations in terms of population distribution functions and note that at equilibrium the ratio $(X Y Z) /(X)(Y Z)$ equals $K_{X, Y Z}^{X Y Z}$, the equilibrium constant. From Eq. (2.6) we have $c_{\mathrm{eq}}(E J) /(X)$ $\times(Y Z)=g_{\text {eq }}(E J)$. Then, upon dividing both sides of Eq. 
(2.18) by the product of the concentrations $(X)(X Y)$, then introducing the equilibrium constant to relate $(X)(Y Z)$ to $(X Y Z)$ and using the definition (2.15) of $F(E J)$, we have

$$
g_{\mathrm{eq}}(E J)=g(E J)+F(E J) K_{X, Y Z}^{X Y Z} \text {. }
$$

Inasmuch as $f(E J)$ is given by Eq. (2.11), it is seen from Eq. (2.19) that the population distribution function $F(E J)$ for the unimolecular dissociation is given by

$$
F(E J)=f(E J) / K_{X, Y Z}^{X Y Z},
$$

a result used in the following.

We consider next for unimolecular reactions the analog of Eq. (2.6) and the subsequent equations. In the steady-state we have $\mathrm{e}^{21}$

$$
\begin{aligned}
0=\frac{d}{d t} F(E J)= & {\left[k_{a}(E J)+\omega\right] F(E J) } \\
& -\iint \omega\left(E^{\prime} J^{\prime}, E J\right) F\left(E^{\prime} J^{\prime}\right) d E^{\prime} d J^{\prime} .
\end{aligned}
$$

That is,

$$
\left[k_{a}(E J)+\omega\right] F(E J)=\iint \omega\left(E^{\prime} J^{\prime}, E J\right) F\left(E^{\prime} J^{\prime}\right) d E^{\prime} d J^{\prime} .
$$

It is seen from Eqs. (2.12) and (2.22) that $f(E J)$ and $F(E J)$ obey the same equation relating values at one $E J$ to those at the other EJ's. Indeed, they should, by virtue of Eq. (2.20).

The unimolecular rate constant $k_{\text {uni }}$ given by Eq. (2.17) thus also equals

$$
k_{\mathrm{uni}}=\iint f(E J) k_{a}(E J) d E d J / K_{X, Y Z}^{X Y Z} .
$$

Thereby,

$$
k_{\mathrm{uni}}=k_{\mathrm{bi}} / K_{X, Y Z}^{X Y Z},
$$

as expected. The $k_{\mathrm{bi}}$ or $k_{\mathrm{uni}}$ can equally be obtained by solving master equations for $f(E J)$ or $F(E J)$, since the latter two functions differ only by a factor $K_{X, Y Z}^{X Y Z}$.

\section{SOLUTION OF THE MASTER EQUATION}

\section{A. Master equation and stepladder model}

In the master equation for $f(E J)$ we first introduce a strong collision approximation for rotation by writing

$$
\omega\left(E^{\prime} J^{\prime}, E J\right)=\omega\left(E^{\prime}, E\right) P_{J}^{\mathrm{eq}},
$$

where $P_{J}^{\mathrm{eq}}$ denotes the equilibrium distribution of $J$ 's. Equation (2.12) now reads

$$
\left[k_{a}(E J)+\omega\right] f(E J)=P_{J}^{\mathrm{eq}} \int \omega\left(E^{\prime}, E\right) f\left(E^{\prime} J^{\prime}\right) d E^{\prime} d J^{\prime} .
$$

We next introduce a stepladder model for the collisional energy transfer, the steps being of energies $E_{n}(n=0$ to $\infty)$ of step size $\Delta E$. We note that in the stepladder model $\langle\Delta E\rangle$ becomes $\Delta E$. If $E_{n}$ is an energy in a deactivation/activation collision stepladder, then $E_{n}=E_{N}-(N-n) \Delta E$, where we choose $N$ as the value corresponding to $E_{N}$ lying in an interval of $\Delta E$ whose lower limit is at the threshold for dissociation. Later we integrate over each $E_{n}$ in its relevant energy interval $\Delta E$. For the distribution function $f\left(E_{n} J\right)$, obtained from Eq. (2.12), Eq. (3.2), and the stepladder model we have

$$
f\left(E_{n} J\right)=\frac{P_{J}^{\mathrm{eq}}\left(\omega_{a, n-1} f_{n-1}+\omega_{d, n+1} f_{n+1}\right)}{k_{a}\left(E_{n} J\right)+\omega},
$$

where

$$
f_{n}=\int f\left(E_{n} J^{\prime}\right) d J^{\prime},
$$

$\omega_{d}$ is the deactivation collision frequency $\omega\left(E_{n^{\prime}} E_{n-1}\right)$, which will be assumed to be independent of $n$ in the region of interest ( $n$ close to $N),{ }^{22}$ and $\omega_{a, n-1}$ is the collision frequency for the reverse (activation) step (the $a$ in $\omega_{a, n-1}$ denotes activation). The two are related by microscopic reversibility, as in Eq. (3.13) given later.

Summed over the steps of energies $E_{0}, E_{1}, \ldots$, the total net incoming flux $S_{J}$ for a given $J$ is

$$
S_{J}=\sum_{n=N}^{\infty} S_{n J}, \quad S_{n J}=k_{a}\left(E_{n}, J\right) f\left(E_{n}, J\right),
$$

the lower summation limit arising since the $k_{a}\left(E_{n} J\right)$ 's vanish for $n<N$. To obtain $k_{\mathrm{bi}}$ one then integrates the $E_{n}$ in each $S_{n J}$ over an energy interval $\Delta E$ and sums (or integrates) over $J$. We then have

$$
k_{\mathrm{bi}}=\sum_{n=N}^{\infty} \iint S_{n J} d E_{n} d J .
$$

Alternatively, $k_{\mathrm{bi}}$ is obtained from the net downward diffusional flux in energy space for $n<N$ via Eq. (3.15) in the following.

A master equation is obtained for the $f$ 's upon integrating Eq. (3.3) over $J$ :

$$
f_{n}=A_{n}\left(\omega_{a, n-1} f_{n-1}+\omega_{d, n+1} f_{n+1}\right),
$$

where

$$
A_{n}=\int_{J}\left[k_{a}\left(E_{n} J\right)+\omega\right]^{-1} P_{J}^{\mathrm{eq}} d J
$$

and $f_{n}$ is defined by Eq. (3.4). This $A_{n}$ becomes $\omega^{-1}$ for $n$ $<N$.

When the master equation (3.7) is solved for the $f_{n}$ 's, subject to the appropriate boundary conditions, $f\left(E_{n} J\right)$ is obtained from Eq. (3.3), and $k_{\mathrm{bi}}$ is then obtained. Two boundary conditions are needed since Eq. (3.7) is a secondorder difference equation. One boundary condition is to take the population distribution function at the lowest step $g_{0}$ to be zero. The second condition is the conservation of flux $S_{J}$. We first show in Sec. III B that the equation relating the $f_{n}$ 's for $n \leqslant N$ can be solved analytically.

\section{B. Flux below the dissociation threshold}

For energies $E_{n}$ below $E_{N}$ the net downward flux $S_{J}^{\text {diff }}$ of the energetic $X Y Z$ 's along the energy stepladder can be written as 


$$
S_{J}^{\mathrm{diff}}=P_{J}^{\mathrm{eq}}\left[\omega_{d} g_{n}-\omega_{a, n-1} g_{n-1}\right] \quad(n \leqslant N),
$$

where

$$
g_{n} \equiv \int g\left(E_{n} J\right) d J
$$

The microscopic reversibility equation is

$$
\begin{aligned}
\omega_{d} g_{n}^{\mathrm{eq}} & \equiv \omega_{d} \int g_{\text {eq }}\left(E_{n}, J\right) d J \\
& \equiv \omega_{a, n-1} \int g_{\text {eq }}\left(E_{n-1}, J\right) d J \\
& \equiv \omega_{a, n-1} g_{n-1}^{\mathrm{eq}},
\end{aligned}
$$

where it can be shown that ${ }^{23}$

$$
P_{J}^{\mathrm{eq}} g_{n}^{\mathrm{eq}}=g_{\text {eq }}\left(E_{n} J\right)=\rho\left(E_{n} J\right) e^{-E_{n} / k_{B} T} / Q_{X, Y Z} \equiv e^{-G_{n}^{J} / k_{B} T} .
$$

The $J$-dependent part of the energy of $X Y Z$ is included in $E_{n}$ and $Q_{X, Y Z}$ is the partition function for $X+Y Z$. [The actual energy of this molecule is $E_{n}+D$, where $D$ is the dissociation energy. To simplify the notation we have denoted this $\rho\left(E_{n}+D, J\right)$ by $\rho\left(E_{n} J\right)$.] In the last equality in Eq. (3.12) we have defined a free energy term $G_{n}^{J}$, a function of $E_{n}$ and $J$.

We next rewrite Eq. (3.11) in terms of the free energy difference, $G_{n-1}^{J}-G_{n}^{J}$ :

$$
\omega_{a, n-1}=\omega_{d} \exp \left(\left(G_{n-1}^{J}-G_{n}^{J}\right) / k_{B} T\right) .
$$

Thereby, using Eq. (3.9),

$$
\begin{aligned}
S_{J}^{\mathrm{diff}}= & P_{J}^{\mathrm{eq}} \omega_{d}\left(g_{n}-g_{n-1} \exp \left(\left(G_{n-1}^{J}-G_{n}^{J}\right) / k_{B} T\right)\right) \\
& (n \leqslant N) .
\end{aligned}
$$

On multiplying Eq. (3.14) by $\exp \left(G_{n}^{J} / k_{B} T\right)$ and summing over $n$ from $n=1$ to $n=N$ we have

$$
S_{J}=P_{J}^{\mathrm{eq}} k_{\mathrm{diff}}^{N}\left(g_{N}-g_{0} \exp \left(\left(G_{0}^{J}-G_{N}^{J}\right) / k_{B} T\right)\right)=P_{J}^{\mathrm{eq}} k_{\mathrm{diff}}^{N} g_{N},
$$

where $k_{\text {diff }}^{N}$ is defined by

$$
k_{\mathrm{diff}}^{N}=\omega_{d} / \sum_{n=1}^{N} \exp \left(\left(G_{n}^{J}-G_{N}^{J}\right) / k_{B} T\right)
$$

and where we have treated the $n=0$ state as a sink by setting $g_{0}=0$. The $P_{J}^{\text {eq }}$ cancels in the difference $G_{n}^{J}-G_{N}^{J}$, and so the $k_{\text {diff }}^{N}$ in Eq. (3.16) does not depend on $J$.

\section{Case of only one reactive step $\mathbf{N}$ in the ladder}

We first consider for simplicity the case where only one recombination step $N$ in the ladder for the $X Y Z$ formation in reaction (2.1) contributes significantly. In this case there is no need to solve the master equation (3.7), since there is only one reactive state $N$ and a solution for all other states $n<N$ was obtained analytically in terms of $g_{N}$, namely, via Eq. (3.15).

The net incident flux $S_{J}$ from reactants $X$ and $Y Z$ for this $E_{N}$ and $J$, using Eqs. (3.5) and (3.3), is given by

$$
S_{J} \equiv S_{N J}=k_{a}\left(E_{N} J\right) f\left(E_{N}, J\right)=\frac{\omega_{a, N-1} P_{J}^{\mathrm{eq}} f_{N-1} k_{a}\left(E_{N} J\right)}{k_{a}\left(E_{N} J\right)+\omega},
$$

where the term $\omega_{d, N+1} f_{N+1}$ in Eq. (3.3) is now absent, since state $N$ is the highest state considered in this one-reactive state model.

Equations (3.9) (for $n=N),(3.15)$, and (3.17) contain the unknowns $g_{N}$ and $g_{N-1}$. Equating these expressions for $S_{J}$ yields two equations. Since there are two unknowns, $g_{N-1}$ and $g_{N}$, one can solve for $g_{N}$. Use of Eq. (3.15) then yields a

$$
g_{N}=g_{N}^{\mathrm{eq}} \omega_{d} k_{a} /\left(\omega_{d} k_{a}+\omega k_{\mathrm{diff}}^{N}\right), \quad k_{a} \equiv k_{a}(E J) .
$$

Further, for this model of only one reactive state $N, \omega_{a, N}$ must vanish and so $\omega$, which equals $\omega_{d}+\omega_{a, N}$, equals $\omega_{d}$. Equations (3.6), (3.15), (3.18) and the first equality in Eq. (3.12), then yield

$$
k_{\mathrm{bi}}=\int_{E_{N}} \int_{J} \frac{k_{a}\left(E_{N} J\right) k_{\mathrm{diff}}^{N} g_{\mathrm{eq}}\left(E_{N} J\right)}{k_{a}\left(E_{N} J\right)+k_{\mathrm{diff}}^{N}} d E_{N} d J,
$$

where the $E_{N}$ integration is over an interval $\Delta E$.

In the strong collision limit $k_{\text {diff }}^{N}$ equals its first term in a series expansion of Eq. (3.16), namely $\omega_{d}$, which in turn now equals $\omega$. Equation (3.19) then reduces to the usual strong collision expression, on taking $\Delta E$ to be very large.

\section{Case of any number of reactive steps}

We consider next the case where there are reactive states $N, N+1, N+2, \ldots \infty$, where $E_{N}$ lies in $(0, \Delta E)$ and $E_{N+n}$ in $[n \Delta E,(n+1) \Delta E]$. The contribution of these particular energies to the flux $S$ at a given $J$ is given by Eq. (3.5), and Eqs. (3.6)-(3.16) also apply. Equations (3.5), (3.3), and (3.7) yield

$$
S_{J}=\sum_{n=N}^{\infty} \frac{k_{a}\left(E_{n} J\right) P_{J}^{\mathrm{eq}} f_{n}}{A_{n}\left[k_{a}\left(E_{n} J\right)+\omega\right]},
$$

noting that $\omega_{d, n+1}$ equals $\omega_{d}$ in the present approximation. The $f_{n}$ 's for $n>N$ can be expressed in terms of $f_{N}$ by solving the master equation (3.7). Upon then equating the $S_{J}$ in Eq. (3.20) to the $S_{J}$ in Eq. (3.15), $g_{N}$ is obtained. The $k_{\mathrm{bi}}$ equals the downward flux $S_{J}$ in Eq. (3.15), integrated over $\Delta E$ and summed (or integrated) over $J$ :

$$
k_{\mathrm{bi}}=\int \sum_{J} P_{J}^{\mathrm{eq}} k_{\mathrm{diff}}^{N} g_{N} d E_{N} .
$$

\section{TWO REACTION EXIT CHANNELS $a, b$}

\section{A. Master equation}

We consider now reactions $(2.1)-(2.4)$ where $Z \neq X$. Equation (2.8) now serves as a definition of a quantity which we again write as $g_{\text {eq }}(E J)$ :

$$
g_{\text {eq }}(E J)=k_{a}^{\prime}(E J) / k_{a}(E J) .
$$

This $g_{\text {eq }}(E J)$ is thereby an equilibrium population distribution function. 
Equation (2.7) is replaced by

$$
\begin{aligned}
0= & k_{a}^{\prime}(E J)-\left[k_{a}(E J)+k_{b}(E J)+\omega\right] g(E J) \\
& +\iint \omega\left(E^{\prime} J^{\prime}, E J\right) g\left(E^{\prime} J^{\prime}\right) d E^{\prime} d J^{\prime},
\end{aligned}
$$

and so with Eqs. (2.8) and (2.11) we then have, instead of Eq. (2.12),

$$
\begin{gathered}
{\left[k_{a}(E J)+k_{b}(E J)+\omega\right] f(E J)-k_{b}(E J) g^{\mathrm{eq}}(E J)} \\
=P_{J}^{\mathrm{eq}} \int \omega\left(E^{\prime}, E\right) f\left(E^{\prime} J^{\prime}\right) d E^{\prime} d J^{\prime},
\end{gathered}
$$

where we have again introduced Eq. (3.1). The bimolecular recombination rate constant for $X+Y Z \rightarrow X Y Z$ is given, following Eqs. (2.11) and (2.13), by

$$
k_{\mathrm{bi}}^{a}=\iint k_{a}(E J) f(E J) d E d J,
$$

where $f(E J)$ now satisfies the master equation (4.3).

Upon introducing the stepladder model for the collisional energy transfer, Eq. (4.3) becomes

$f\left(E_{n} J\right)=\frac{P_{J}^{\mathrm{eq}}\left(\omega_{a, n-1} f_{n-1}+\omega_{d} f_{n+1}\right)+k_{b}\left(E_{n} J\right) g^{\mathrm{eq}}\left(E_{n} J\right)}{k_{a}\left(E_{n} J\right)+k_{b}\left(E_{n} J\right)+\omega}$,

where $f_{n}$ is again given by Eq. (3.4). The bimolecular rate constant is again obtained from the downward diffusional probability flux given by Eq. (3.5), integrated over $E_{N}$ and summed over $J$. Thereby, the bimolecular rate constant, which we now denote by $k_{\mathrm{bi}}^{a}$ to indicate that the entrance channel in the example is $a$, is again given by Eq. (3.6), namely

$$
k_{\mathrm{bi}}^{a}=\sum_{J} \int P_{J}^{\mathrm{eq}} k_{\mathrm{diff}}^{N} g_{N} d E_{N} .
$$

A master equation for the $f_{n}$ 's obtained by integrating $f\left(E_{n} J\right)$ in Eq. (4.4) over $J$, is now

$$
f_{n}=B_{n}\left(\omega_{a, n-1} f_{n-1}+\omega_{d} f_{n+1}\right)+C_{n},
$$

where

$$
\begin{aligned}
& B_{n}=\int \frac{P_{J}^{\mathrm{eq}} d J}{k_{a}\left(E_{n} J\right)+k_{b}\left(E_{n} J\right)+\omega}, \\
& C_{n}=\int \frac{k_{b}\left(E_{n} J\right) g^{\mathrm{eq}}\left(E_{n} J\right) d J}{k_{a}\left(E_{n} J\right)+k_{b}\left(E_{n} J\right)+\omega} .
\end{aligned}
$$

We note that the two exit channels will have slightly different energy thresholds, because of a difference in zero-point energies of $Y Z$ and $X Y$, and the integration in Eq. (4.6) over $E_{N}$ is intended to begin at the lower threshold of the two. The master equation (4.7) is first solved for the $f$ 's in terms of $f_{N}$ and hence of $g_{N}$. Then, as before, $g_{N}$ can be obtained from the boundary condition describing flux conservation. We proceed as follows.

The net flux entering via channel $a$ for a given $J, S_{J}^{a}$, is the same as Eq. (3.5) and so we have

$$
S_{J}^{a}=\sum_{n=N}^{\infty} S_{n J}^{a}, \quad S_{n J}^{a}=k_{a}\left(E_{n}, J\right) f\left(E_{n} J\right) .
$$

The flux outgoing in channel $b, S_{J}^{b}$, is given by

$$
S_{J}^{b}=\sum_{n=N}^{\infty} S_{n J}^{b}, \quad S_{n J}^{b}=k_{b}\left(E_{n}, J\right) g\left(E_{n} J\right) .
$$

The downward diffusional flux $S_{J}^{\text {diff }}$ is again given by Eq. (3.15) and conservation of flux yields

$$
S_{J}^{a}=S_{J}^{b}+S_{J}^{\mathrm{diff}}
$$

which serves as a boundary condition for solution of the master equation, by providing an equation for $g_{N}$. Equation (4.6) then yields $k_{\mathrm{bi}}^{a}$.

This material balance equation (4.11) can also be written equivalently as

$$
\begin{gathered}
\sum_{n=N}^{\infty}\left[k_{a}\left(E_{n} J\right)+k_{b}\left(E_{n} J\right)+k_{\mathrm{diff}}^{N}\right] g\left(E_{n} J\right) \\
=\sum_{n=N}^{\infty} k_{a}\left(E_{n} J\right) g^{\mathrm{eq}}\left(E_{n} J\right) .
\end{gathered}
$$

The present equations, or their equivalent, are utilized elsewhere in a treatment of isotopic effects. ${ }^{12,13}$

\section{B. Special case of one reactive step $\boldsymbol{N}$}

An expression for $g_{N}$ is obtained from Eqs. (3.9), (3.12), (3.15), (4.5), and (4.9)-(4.11). From Eq. (4.6) and the first equality in (3.12) one then obtains

$$
k_{\mathrm{bi}}^{a}=\int_{E_{N}} \int_{J} \frac{k_{a}\left(E_{N} J\right) k_{\mathrm{diff}}^{N} g_{\mathrm{eq}}(E J)}{k_{a}\left(E_{N} J\right)+k_{b}\left(E_{N} J\right)+k_{\mathrm{diff}}^{N}} d E_{N} d J
$$

On letting $\Delta E$ become very large $k_{\text {diff }}^{N}$ reduces to $\omega_{d}$ and hence to $\omega$ as before, and the result obtained in Ref. 18 is recovered. Further, Eq. (4.13) reduces to Eq. (3.22) when $k_{b}\left(E_{N} J\right)$ vanishes. The equation for $k_{\mathrm{bi}}^{b}$ is obtained from Eq. (4.13) by interchanging $a$ 's and $b$ 's.

\section{ISOTOPIC EXCHANGE REACTIONS}

The isotopic exchange reactions

$$
X+Y Z \rightarrow X Y+Z,
$$

where $X, Y, Z$ are isotopes, are typically studied at low pressures, where the newly formed vibrationally hot $X Y Z^{*}$ typically dissociates before it can undergo a collision with the bath gas. The results are then independent of whether the deactivating collisions are strong or weak, and so the equations given in Refs. 18 and 19 apply.

\section{ACKNOWLEDGMENT}

It is a pleasure to acknowledge the support of this research by the National Science Foundation.

\footnotetext{
${ }^{1}$ R. G. Gilbert and S. C. Smith, Theory of Unimolecular and Recombination Reactions (Blackwell Scientific, Boston, 1990), and references cited therein. Section (6.10) for unimolecular reactions prompted the $J$-averaging used in Eq. (3.2).

${ }^{2}$ K. A. Holbrook, M. J. Pilling, and S. H. Robertson, Unimolecular Reactions (Wiley, New York, 1996), 2nd ed., and references cited therein.

${ }^{3}$ T. Baer and W. L. Hase, Unimolecular Reaction Dynamics, Theory and
} 
Experiments (Oxford University Press, New York, 1996), and references therein.

${ }^{4}$ D. M. Wardlaw and R. A. Marcus, Adv. Chem. Phys. 70, 231 (1988).

${ }^{5}$ S. J. Klippenstein, in The Chemical Dynamics and Kinetics of Small Radicals, Part I, edited by K. Liu and A. Wagner (World Scientific, Singapore, 1995), and references therein.

${ }^{6}$ D. C. Tardy and B. S. Rabinovitch, J. Chem. Phys. 48, 1282 (1968).

${ }^{7}$ D. C. Tardy and B. S. Rabinovitch, J. Chem. Phys. 45, 3720 (1966).

${ }^{8}$ I. Oref and D. C. Tardy, Chem. Rev. 90, 1407 (1990).

${ }^{9}$ H. G. Löhmannsröben and K. Luther, Chem. Phys. Lett. 144, 173 (1988).

${ }^{10}$ A. Pashutski and I. Oref, J. Phys. Chem. 92, 178 (1988).

${ }^{11}$ Reference 2, Chap. 9.

${ }^{12}$ Y.-Q. Gao and R. A. Marcus (unpublished).

${ }^{13}$ Y.-Q. Gao and R. A. Marcus (unpublished)

${ }^{14}$ H. Hippler, R. Rahn, and J. Troe, J. Chem. Phys. 93, 6560 (1990); Ref. 2, Chap. 7, and references cited therein.

${ }^{15}$ See, M. H. Thiemens and T. Jackson, Geophys. Res. Lett. 17, 717 (1990).

${ }^{16}$ See, K. Mauersberger, B. Erbacher, D. Krankowsky, J. Günther, and R. Nickel, Science 283, 370 (1999).

${ }^{17}$ M. R. Wiegell, N. W. Larsen, T. Pedersen, and H. Egsgaard, Int. J. Chem. Kinet. 29, 745 (1997).

${ }^{18}$ B. C. Hathorn and R. A. Marcus, J. Chem. Phys. 111, 4087 (1999).

${ }^{19}$ B. C. Hathorn and R. A. Marcus, J. Chem. Phys. 113, 9497 (2000).
${ }^{20}$ There is very little difference whether one sets $d g(E J) / d t=0$, as in Eq. (2.7), or sets $d c(E J) / d t=0$. This fact is most easily seen for the unimolecular reaction and we return to this point in Ref. 21.

${ }^{21}$ Given $d F(E J) / d t=0$ in Eq. (2.21), we can explore its consequences for $d C(E J) / d t$. We have, using Eq. (2.15), $0=-\left[C(E J) /(X Y Z)^{2}\right] d(X Y Z) /$ $d t+(d C(E J) / d t) /(X Y Z)$. But the first term equals $-[C(E J) /$ $(X Y Z)] k_{\text {uni }}$, i.e., $k_{\text {uni }} F_{E J}$, which is typically small relative to the individual terms enhancing or decreasing $F(E J)$ in the rate expression (2.21). Accordingly, $d C(E J) / d t$ typically equals zero to a good approximation.

${ }^{22}$ The total collision frequency $\omega$ of an ozone molecule in state $n$ is $\omega_{d}$ $+\omega_{a, n}+\omega_{0}$, where the 0 label indicates the possibility that there is no net energy transfer. We note in passing that the approximation that in the neighborhood of $n=N, \omega_{d}$ can be treated as constant while $\omega_{a, n}$ obeys Eq. (3.4) can have some implications for the behavior of $\omega$.

${ }^{23}$ We have $P_{J}^{\text {eq }} g_{n}^{\text {eq }}=P_{J}^{\text {eq }} \int g_{\text {eq }}\left(E_{n}, J^{\prime}\right) d J^{\prime}$. We write $g_{\text {eq }}\left(E_{n}, J^{\prime}\right)$ as $\left(2 J^{\prime}\right.$ +1) $\rho_{\mathrm{vib}}\left(E_{n}-E_{J^{\prime}}\right) C$, where $C=\exp \left(-E_{n} / k_{B} T\right) / Q_{X, Y Z}$. Here, $E_{n}-E_{J^{\prime}}$ (in turn, a shorthand for $\left.D+E_{n}-E_{N}-E_{J^{\prime}}\right)$ is the vibrational energy $E_{\mathrm{vib}}^{n}$, and so $\rho_{\mathrm{vib}}\left(E_{n}-E_{J^{\prime}}\right)$ is the vibrational density of states. Then writing $E_{n}$ as $E_{n}-E_{J^{\prime}}+E_{J^{\prime}}$ and integrating over $J^{\prime}$ one obtains $P_{J}^{\mathrm{eq}} \rho_{\mathrm{vib}}\left(E_{\mathrm{vib}}^{n}\right)$ $\times \exp \left(-E_{\mathrm{vib}}^{n} / k T\right) / Q_{X, Y Z}$, which can be rewritten as $\rho\left(E_{n}, J\right)$ $\times \exp \left(-E_{n} / k_{B} T\right) / Q_{X, Y Z}$, i.e., as $g_{\text {eq }}\left(E_{n}, J\right)$, as in the text. 